\title{
Terahertz heterodyne receiver based on a quantum cascade laser and a superconducting bolometer
}

\author{
J. R. Gao ${ }^{\text {a),b) }}$ and J. N. Hovenier \\ Kavli Institute of NanoScience, Faculty of Applied Sciences, Delft University of Technology, \\ Lorentzweg 1, 2628 CJ, Delft, The Netherlands \\ Z. Q. Yang, J. J. A. Baselmans, and A. Baryshev \\ SRON National Institute for Space Research, Sorbonnelaan 2, 3584 CA, Utrecht, The Netherlands \\ M. Hajenius, ${ }^{\text {a) }}$ T. M. Klapwijk, A. J. L. Adam, and T. O. Klaassen \\ Kavli Institute of NanoScience, Faculty of Applied Sciences, Delft University of Technology, Lorentzweg 1, \\ 2628 CJ, Delft, The Netherlands \\ B. S. Williams, S. Kumar, and Q. Hu \\ Department of Electrical Engineering and Computer Science and Research Laboratory of Electronics, \\ Massachusetts Institute of Technology, Cambridge MA 02139 \\ J. L. Reno \\ Sandia National Laboratories, Albuquerque, New Mexico 87185-0601
}

(Received 22 February 2005; accepted 11 May 2005; published online 8 June 2005)

\begin{abstract}
We report the first demonstration of an all solid-state heterodyne receiver that can be used for high-resolution spectroscopy above $2 \mathrm{THz}$ suitable for space-based observatories. The receiver uses a $\mathrm{NbN}$ superconducting hot-electron bolometer as mixer and a quantum cascade laser operating at $2.8 \mathrm{THz}$ as local oscillator. We measure a double sideband receiver noise temperature of $1400 \mathrm{~K}$ at $2.8 \mathrm{THz}$ and $4.2 \mathrm{~K}$, and find that the free-running QCL has sufficient power stability for a practical receiver, demonstrating an unprecedented combination of sensitivity and stability. (C) 2005 American Institute of Physics. [DOI: 10.1063/1.1949724]
\end{abstract}

Present day heterodyne receivers use a combination of an electronically tunable solid-state local oscillator (LO) source based on multiplier chains, ${ }^{1}$ with either a superconductor-insulator-superconductor ${ }^{2}$ mixer or a hotelectron bolometer (HEB) mixer. ${ }^{3,4}$ The heterodyne instrument for the far infrared on the Herschel Space Observatory, ${ }^{5}$ to be launched in 2007, is the first instrument to perform very high-resolution spectroscopy using such receivers from $480 \mathrm{GHz}$ to $1.9 \mathrm{THz}$ in space. Future space missions require improved angular resolution, improved sensitivity, and, most important, an increase in frequency from 2 to $6 \mathrm{THz}{ }^{6}$ The development of new receivers operating at such high frequencies is limited by the availability of suitable LO sources. The existing solid state LOs are unlikely to generate sufficient output power at such high frequencies since the power falls off rapidly with increasing frequency due to reduced multiplication efficiency. ${ }^{1}$ Optically pumped gas lasers can operate at higher frequencies but are in general massive, bulky, and power hungry. Very recently, a new type of solidstate $\mathrm{THz}$ source was developed based on quantum cascade laser (QCL) structures. ${ }^{7}$ This new source holds great promise for LO applications because of its compactness and high power efficiency. Here we report the first demonstration of a fully operational heterodyne receiver at $2.8 \mathrm{THz}$ based on such a THz QCL as LO source and a hot-electron bolometer as mixing element.

The concept of a QCL was first demonstrated in the midinfrared $(\lambda \cong 4 \mu \mathrm{m} ; 75 \mathrm{THz})$ by Faist et $a l .{ }^{8}$ Photons are cre-

\footnotetext{
${ }^{a)}$ Also with SRON National Institute for Space Research, Utrecht, The Netherlands.

${ }^{b)}$ Electronic mail: j.r.gao@tnw.tudelft.nl
}

ated via electronic intersubband transitions in semiconductor heterostructures that take place entirely within the conduction band. Furthermore, in a QCL the heterostructure active region consists of a stack of repeated identical quantum well modules (typically 20-200), which enables a single electron to cascade down and emit a photon in each module. Due to this cascading effect, QCLs have large quantum efficiency and high output power. The QCL frequency range is determined by the energy spacing of the subbands, which is set by the design and growth of the quantum-well structure. The precise operating frequency is determined by the waveguide cavity of the laser. While the development of a THz QCL has proven to be more challenging than for mid-infrared QCLs because of the difficulty of achieving population inversion for small subband separations and of obtaining a low-loss cavity for long wavelengths, a first THz QCL was demonstrated at $4.4 \mathrm{THz}$ by Köhler et al. ${ }^{7}$ To be suitable as an LO, a QCL has to meet a number of essential requirements, such as single line lasing with a high spectral purity, cw operation, adequate output power, and good stability.

We use a QCL device described in Ref. 9, which is based on resonant phonon scattering to selectively depopulate the lower radiation level, while maintaining a long upperlevel lifetime. The active region contains 176 GaAs $/ \mathrm{Al}_{0.15} \mathrm{Ga}_{0.85} \mathrm{As}$ quantum-well modules, with a total thickness of $10 \mu \mathrm{m}$. The cavity of the QCL is a double-sided metal-metal waveguide fabricated via $\mathrm{Cu}-\mathrm{Cu}$ thermocompression wafer bonding, which provides low-loss mode confinement at $\mathrm{THz}$ frequencies. Similar QCLs have allowed lasing at lower frequencies down to $2.1 \mathrm{THz}$ (see Ref. 10) and in cw mode up to $93 \mathrm{~K},{ }^{11}$ and tuning the frequency by $12 \mathrm{GHz}$ by varying temperature. ${ }^{12}$ The $25 \mu \mathrm{m}$ wide ridge 


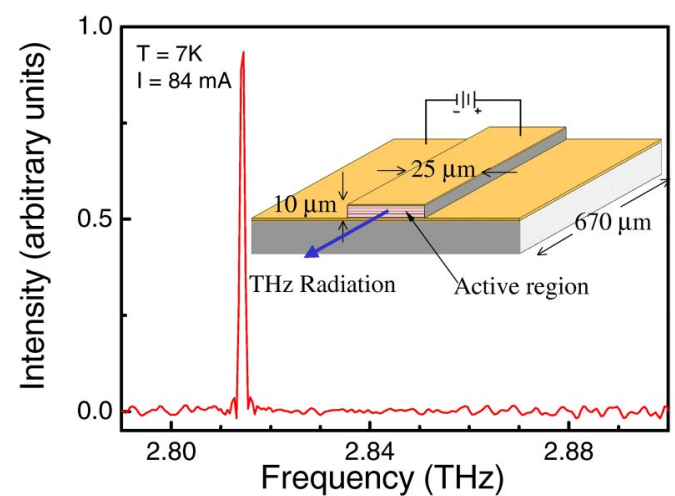

FIG. 1. (Color online) Emission spectrum of the quantum cascade laser biased with a dc current $\mathrm{I}$ of $84 \mathrm{~mA}$ and operated at a temperature $\mathrm{T}$ around $7 \mathrm{~K}$. The inset shows a schematic view of the QCL and the dimensions of the laser cavity.

waveguide was cleaved at both ends to form a $670 \mu \mathrm{m}$ long Fabry-Perot cavity (shown schematically in the inset of Fig. 1). The QCL is indium soldered to a copper mount and is attached to the cold plate of a helium vacuum Dewar. The main figure shows a typical spectrum of the QCL lasing at $2.814 \mathrm{THz}$ (wavelength $\lambda_{0}=106.6 \mu \mathrm{m}$ ), measured using a Fourier-transform spectrometer (FTS). It is biased with a dc current of $84 \mathrm{~mA}$ and dissipates a power of about $1 \mathrm{~W}$. When operated in $\mathrm{cw}$ and free-running mode, a single lasing line is observed, with a linewidth of about $1 \mathrm{GHz}$, which is limited by the resolution of the FTS. The actual linewidth is likely to be much smaller, for example, 30 (see Ref. 13) and $65 \mathrm{kHz}$ (obtained over an arbitrarily long period of time using frequency/phase locking). ${ }^{14}$ The maximum output power is $1 \mathrm{~mW}$, measured using a Winston cone in front of the QCL to collect all the radiated power. In a practical heterodyne experiment, the available power will be limited by the divergence of the beam, the window size, and the distance between the QCL and the first focusing element of the optics.

In recent years, phonon-cooled HEB mixers ${ }^{15,16}$ have matured as the only sensitive mixer for the frequency range from 1.5 to $6 \mathrm{THz}$. It uses the response to radiation of the temperature-dependent resistance of a small $\mathrm{NbN}$ superconducting bridge. ${ }^{3}$ The electrons (hot electrons) in the superconducting bridge are heated by the $\mathrm{THz}$ photons (predominantly by the LO signal) and the local electron temperature reaches the critical temperature of the superconductor. The mixing signal at intermediate frequency (IF) is the result of the fact that the electron temperature can follow the beatfrequency of the LO signal and the signal to be detected. The sensitivity of receivers is characterized by their receiver noise temperature $\left(T_{N, \text { rec }}\right)$ in Kelvin. The HEB receivers have demonstrated superior sensitivity; for example, a $T_{N, \text { rec }}$ of $950 \mathrm{~K}$ at $2.5 \mathrm{THz} .{ }^{16}$

Figure 2 shows a schematic view of the experimental setup with the QCL and the HEB mounted in two separate dewars. A wideband spiral antenna coupled NbN HEB mixer ${ }^{17}$ is used with a superconducting bridge of $4 \mu \mathrm{m}$ wide, $0.4 \mu \mathrm{m}$ long, and about $4 \mathrm{~nm}$ thick. The normal state resistance $R_{N}$ of the device, measured above the critical temperature of $9 \mathrm{~K}$, is $65 \Omega$. Without radiation applied a critical current $I_{c}$ of $320 \mu \mathrm{A}$ is observed at $4.2 \mathrm{~K}$. The radiation is coupled to the antenna using a standard quasi-optical technique: the Si chip with the HEB is glued to the back of an elliptical, antireflection-coated Si lens. The lens is placed in a

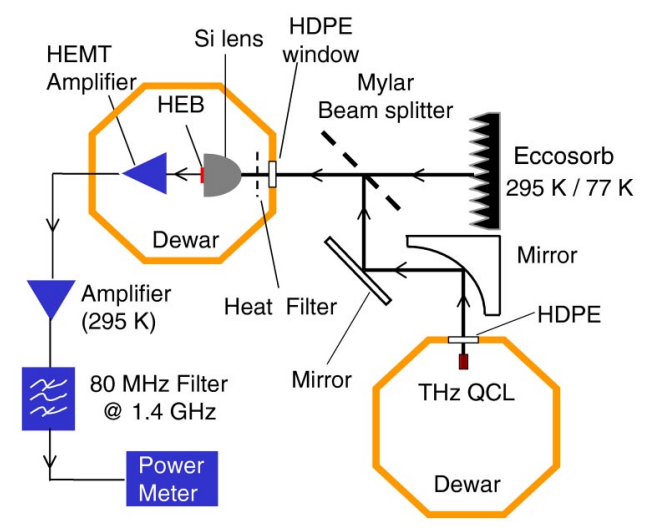

FIG. 2. (Color online) Schematic view of the heterodyne measurement setup.

metal mixer block, thermally anchored to the $4.2 \mathrm{~K}$ cold plate. When used with a gas laser at $2.5 \mathrm{THz}$ a $T_{N, \text { rec }}$ is obtained of $1200 \mathrm{~K},{ }^{18}$ using a setup identical to that in the experiment with the QCL.

The divergent beam from the QCL passes through a high-density polyethylene (HDPE) Dewar window and is collimated with a parabolic mirror. The radiation is further guided to the HEB Dewar through a flat mirror and a $6 \mu \mathrm{m}$ thick Mylar ${ }^{\mathrm{TM}}$ beam splitter. A blackbody source (of Eccosorb $^{\circledR}$ ) is used as the signal, which defines a hot load at $295 \mathrm{~K}$ and a cold load at $77 \mathrm{~K}$. The signal is combined with the QCL beam through the beam splitter. Both signals pass through the thin HDPE window and a metal mesh heat filter at $77 \mathrm{~K}$ of the HEB Dewar. The total known loss is $-16.2 \mathrm{~dB}$ for the QCL (to the HEB) optical path and $-4.0 \mathrm{~dB}$ for the hot/cold load path. The IF signal, resulting from the mixing of the LO and the hot/cold load signal, is amplified using a low noise amplifier operated at $4.2 \mathrm{~K}$, and is further fed to a room-temperature amplifier and filtered at $1.4 \mathrm{GHz}$ in a band of $80 \mathrm{MHz}$. The entire IF chain has a gain of $80 \mathrm{~dB}$ and a noise temperature of $4 \mathrm{~K}$.

The key result of this work is demonstrated in Fig. 3. A set of current versus voltage $(I-V)$ curves of the HEB is shown for various levels $(270,300$, and $330 \mathrm{nW})$ of the effective power of radiation absorbed at the HEB, together with the receiver noise temperature, $T_{N, \text { rec }}$, as a function of voltage. (The inset shows a top view of the HEB with its

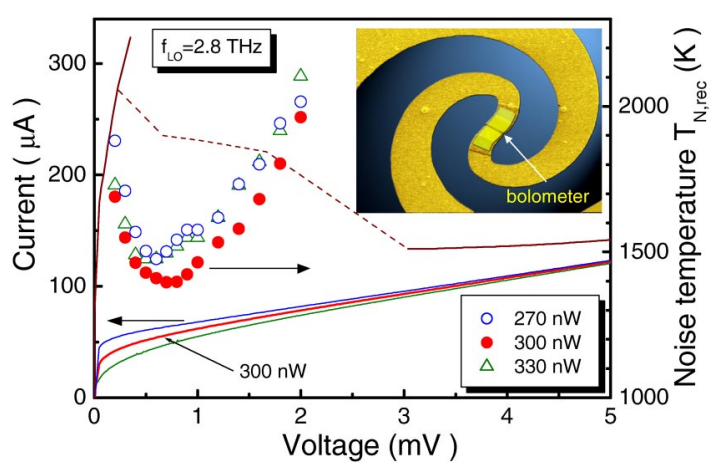

FIG. 3. (Color online) Current-voltage characteristics (full lines, left axis) of a $\mathrm{NbN}$ hot electron bolometer (HEB) mixer without and with radiation from the QCL at $2.814 \mathrm{THz}$. The dashed line indicates an unstable bias region. The measured receiver noise temperature $T_{N, \text { rec }}$ (symbols, right axis) versus the bias voltage for different LO power levels at the HEB. The inset shows a top view of the HEB with its spiral antenna. 


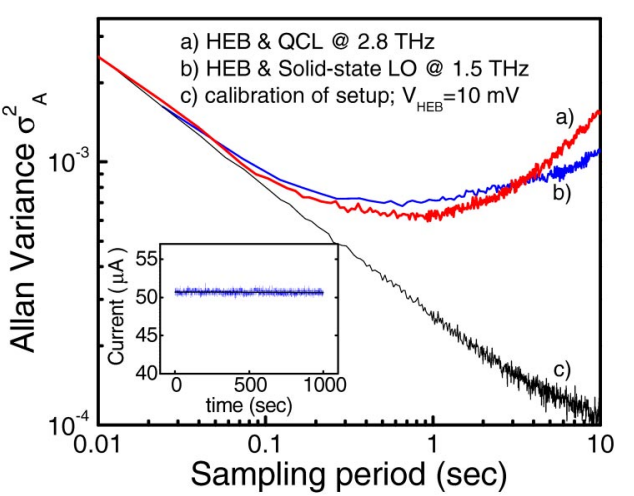

FIG. 4. (Color online) Allan variance $\sigma_{\mathrm{A}}^{2}$ of the normalized output power of the HEB as a function of the sampling period: (a) for the HEB-QCL receiver at optimum operating point, (b) for a similar HEB mixer using a phaselocked solid-state LO at $1.52 \mathrm{THz}$, and (c) for the receiver when the HEB is biased at $10 \mathrm{mV}$. The inset shows the current of the HEB at optimum operating point as a function of time.

spiral antenna.) The power level, which is estimated at the HEB by the isothermal technique, ${ }^{19}$ is varied by changing the dc bias current of the QCL. The noise temperature $T_{N \text {,rec }}$ is determined from the ratio of the IF output noise power for a hot and a cold load. ${ }^{18}$ Each set of $T_{N, \text { rec }}-V$ data shows a minimum region, indicating the optimum bias point. Best results are obtained for $300 \mathrm{nW}$ LO power and $0.7 \mathrm{mV} \mathrm{dc}$ bias with $T_{N \text {,rec }}$ being as low as $1400 \mathrm{~K}$, comparable to what was obtained with the gas laser at the lower frequency of $2.5 \mathrm{THz}$. The maximum power from the QCL coupled to the HEB, taking all losses into account, is estimated to be $14 \mu \mathrm{W}$. This is only $1.4 \%$ of the total output power available from the QCL, which is due to the poor optical coupling of the divergent beam.

We have performed two separate experiments to determine the stability of the HEB-QCL receiver, in which the HEB is operated at the point where it gives the lowest noise temperature. First, we measured the Allan variance $\sigma^{2}{ }_{\mathrm{A}}(\tau)$ of the normalized IF output power, given ${ }^{20}$ by $\sigma^{2}{ }_{\mathrm{A}}(\tau) \equiv \frac{1}{2} \sigma(\tau)^{2}$, where $\sigma^{2}$ is the average squared standard deviation of each number from its mean and $\tau$ is the sampling period. The noise of any receiver is a combination of three terms: white (uncorrelated) noise, $1 / f$ electronic noise, and low-frequency drift. Since, to first order, only white noise can be integrated out, there is an optimum integration time, known as the "Allan" time $T_{\mathrm{A}}$, beyond which the signal/noise ratio no longer improves. ${ }^{21} \mathrm{~A}$ measurement of $\sigma_{\mathrm{A}}^{2}(\tau)$ is a powerful tool to distinguish the various noise terms in a real receiver and to evaluate its optimal integration time. The measured $\sigma^{2}{ }_{\mathrm{A}}(\tau)$ for the HEB-QCL receiver is plotted as a function of the sampling period in Fig. 4. As a comparison, we also plot $\sigma_{\mathrm{A}}^{2}(\tau)$ obtained using a similar HEB mixer pumped by a phase-locked solid-state LO at $1.5 \mathrm{THz} .{ }^{1}$ Both measurements indicate an identical $T_{\mathrm{A}}$ of about $0.5 \mathrm{~s}$ within the $80 \mathrm{MHz}$ bandwidth of the IF chain. In the same figure we also include the measured $\sigma_{\mathrm{A}}^{2}(\tau)$ when the HEB is biased at $10 \mathrm{mV}$, which shows the expected white noise behavior. This suggests that the observed Allan time is limited by the HEB mixer itself and not by any other sources, such as mixer bias, amplifier fluctuations, etc. Secondly, we have measured the output power of the QCL as a function of time using the HEB as a direct detector. As shown in the inset of Fig. 4, the averaged current of the HEB varies only $0.2 \%$ in a period of $1000 \mathrm{~s}$, indicating that the output power of a QCL is sufficiently stable over a long time scale.

In conclusion, we have demonstrated that a heterodyne receiver based on a QCL and an HEB can be operated with an excellent receiver noise temperature of $1400 \mathrm{~K}$ at $2.8 \mathrm{THz}$, uncorrected for losses in the optics. The freerunning QCL has sufficient power stability for a practical receiver and the output power is more than adequate for use with HEBs. We envision the QCLs, the HEBs, and other components to be integrated together into a mechanical cryocooler at $4 \mathrm{~K}$, leading to a compact, easy to use, rugged, and space qualifiable all-solid-state receiver system.

We acknowledge J.W. Kooi for his advice on the Allan variance measurement, and G.N. Gol'tsman and B. Voronov for providing the $\mathrm{NbN}$ films. The work in NL is supported by RadioNet and ESA. The work at MIT is supported by AFOSR, NASA, and the NSF. Sandia is a multiprogram laboratory operated by Sandia Corporation, a Lockheed Martin Company, for the US Dept. of Energy under Contract DE-AC04-94AL85000.

${ }^{1}$ I. Mehdi, E. Schlecht, G. Chattopadhyay, and P. H. Siegel, Proc. SPIE 4855, 435 (2003).

${ }^{2}$ B. D. Jackson, A. M. Baryshev, G. de Lange, J. R. Gao, S. V. Shitov, N. N. Iosad, and T. M. Klapwijk, Appl. Phys. Lett. 79, 436 (2001).

${ }^{3}$ E. M. Gershenzon, G. N. Gol'tsman, I. G. Gogidze, A. I. Eliantev, B. S. Karasik, and A. D. Semenov, Sov. Phys. Superconductivity 3, 1582 (1990).

${ }^{4}$ S. Cherednichenko, P. Khosropanah, E. Kollberg, M. Kroug, and H. Merkel, Physica C 372-376, 407 (2002).

${ }^{5} \mathrm{http} / / /$ sci.esa.int/science-e/www/area/index.cfm?fareaid $=16$

${ }^{6}$ Th. de Graauw, J. Cernicharo, W. Wild, A. Bos, J-W. den Herder, A. Gunst, F. Helmich, B. Jackson, H-J. Langevelde, P. Maat, J. MartinPintado, J. Noordam, A. Quirrenbach, P. Roelfsema, L. Venema, P. Wesselius, and P. Yagoubov, Proc. SPIE 5487, 1522 (2004).

${ }^{7}$ R. Köhler, A. Tredicucci, F. Beltram, H. E. Beere, E. H. Linfield, A. G. Davies, D. A. Ritchie, R. C. Iotti, and F. Rossi, Nature (London) 417, 156 (2002).

${ }^{8}$ J. Faist, F. Capasso, D. L. Sivco, C. Sirtori, A. L. Hutchinson, and A. Y. Cho, Science 264, 553 (1994).

${ }^{9}$ Q. Hu, B. S. Williams, S. Kumar, H. Callebaut, S. Kohen, and J. L. Reno (unpublished).

${ }^{10}$ B. S. Williams, S. Kumar, Q. Hu, and J. L. Reno, Electron. Lett. 40, 431 (2004).

${ }^{11}$ S. Kumar, B. S. Williams, S. Kohen, Q. Hu, and J. L. Reno, Appl. Phys. Lett. 84, 2494 (2004).

${ }^{12}$ B. S. Williams (unpublished).

${ }^{13}$ A. Barkan, F. K. Tittel, D. M. Mittleman, R. Dengler, P. H. Siegel, G. Scalari, L. Ajili, J. Faist, H. E. Beere, E. H. Linfield, A. G. Davies, and D. A. Ritchie, Opt. Lett. 29, 575 (2004).

${ }^{14}$ A. L. Betz, R. T. Boreiko, B. S. Williams, S. Kumar, and Q. Hu (unpublished).

${ }^{15}$ A. D. Semenov, H.-W. Hübers, J. Schubert, G. N. Gol'tsman, A. I. Elantiev, B. M. Voronov, and E. M. Gershenzon, J. Appl. Phys. 88, 6758 (2000).

${ }^{16}$ J. J. A. Baselmans, M. Hajenius, J. R. Gao, T. M. Klapwijk, P. A. J. de Korte, B. Voronov, and G. Gol'tsman, Appl. Phys. Lett. 84, 1958 (2004).

${ }^{17}$ M. Hajenius, J. J. A. Baselmans, J. R. Gao, T. M. Klapwijk, P. A. J. de Korte, B. Voronov, and G. Gol'tsman, Supercond. Sci. Technol. 17, S224 (2004). We use the device in which the contacts have a thin $\mathrm{Nb}$ interlayer.

${ }^{18}$ We use the Callen-Welton definition for the double sideband receiver noise temperature, as described in: A. R. Kerr, IEEE Trans. Microwave Theory Tech. 47, 325 (1999).

${ }^{19}$ H. Ekström, B. S. Karasik, E. L. Kollberg, and K. S. Yngvesson, IEEE Trans. Microwave Theory Tech. 43, 938 (1995).

${ }^{20}$ D. W. Allan, Proc. IEEE 54, 221 (1996).

${ }^{21}$ J. W. Kooi, G. Chattopadhyay, M. Thielman, T. G. Phillips, and R. Schieder, Int. J. Infrared Millim. Waves 21, 689 (2000). 